\title{
Numerical linear algebra in the integrity theory of the Global Positioning System is
}

\author{
Xiao-Wen Chang*, Christopher C. Paige \\ School of Computer Science, McGill University, 3480 University Street, Montreal, Quebec, Canada
}

H3A $2 A 7$

\begin{abstract}
The Global Positioning System (GPS) is a satellite based navigation system. Since safety is the main concern for aircraft navigation, various means of monitoring the integrity (certainty of position) have been developed. This is an important area of research in the GPS community. In the following, it will be shown how some numerical linear algebra techniques can be applied to this interesting application. A typical model is presented. A uniform approach to derive the statistics for fault detection and isolation by orthogonal transformations is given. It is shown that the diagonal elements $\omega_{i i}^{2}$ of the orthogonal projection matrix onto the residual space are fundamental to the theory and understanding of integrity. $\omega_{i i}$ can, for example, have a drastic effect on integrity when they are small. The sensitivity of related problems in this area are discussed. (c) 2002 Elsevier Science B.V. All rights reserved.
\end{abstract}

Keywords: Global Positioning System; Integrity theory; Test statistics; Orthogonal transformations; Sensitivity

\section{Introduction}

The Global Positioning System (GPS) is an all-weather, worldwide, continuous coverage, satellite-based navigation system. GPS satellites transmit signals that allow one to determine, with great accuracy, the location of GPS receivers (see, for example, Hofmann-Wellenhof et al., 1997; Parkinson et al., 1996 and Strang and Borre, 1997). As safety is the main concern for aircraft, one must find ways of ensuring that aircraft

\footnotetext{
it This research was supported by NSERC of Canada Grant RGPIN217191-99 and FCAR of Quebec, Canada Grant 2001-NC-66487 for Xiao-Wen Chang, and by NSERC of Canada Grant OGP0009236 for Christopher C. Paige.

* Corresponding author.

E-mail addresses: chang@cs.mcgill.ca (X.-W. Chang), paige@cs.mcgill.ca (C.C. Paige).
} 
can safely utilize the GPS system. Even though satellite anomalies are rare, when they do occur, it is very likely that several hundred aircraft will be affected simultaneously. Therefore, means of integrity monitoring have to be developed. Navigation system integrity refers to the ability of a system to provide a timely warning to users when the system should not be used for navigation. Currently, the main approach is the receiver autonomous method, referred to simply as receiver autonomous integrity monitoring (RAIM). RAIM has two functions: one is to detect failures in the input data, and if possible, isolate the failed data; the other is to estimate the maximum error radial that the navigation solution may produce, see Brenner (1990), Chin et al. (1992-93), Kelly (1998), Lee et al. (1996), Parkinson and Axelrad (1988), and Sturza (1988-89) and others.

In this paper, we show how numerical linear algebra techniques can be applied to this important RAIM area. Specifically, we will use orthogonalization techniques to give a very uniform approach to deriving the statistics for fault detection and isolation that are used throughout the literature, and show how certain scalars $\omega_{i i}$ related to a projection matrix are fundamental to the area, and how they affect the integrity when they are small. Also, we discuss the sensitivity of related problems in this area.

This paper is organized as follows. In Section 2 we describe the basic properties of the general linear model with possible faults that we will use, and also we introduce some background in GPS integrity. In Section 3 we consider deleting an observation from the linear model, leading to a reduced model, and the effect of this on the residual and parity vectors. We will discuss the error covariance matrix of the reduced model and show that the scalars $\omega_{i i}$ have damaging effects when they are small. In Section 4 key statistics for fault detection and isolation are derived in a uniform way. In Section 5 we will discuss the sensitivity of the statistics. Finally, we give a brief summary in Section 6.

We work with reals only, and use $i, j, m$ and $n$ to denote integers, other lower case Roman letters to denote vectors, lower case Greek letters to denote scalars, and upper case Roman to denote matrices. Superscript $\mathrm{T}$ will denote transpose, and $A^{\dagger}$ is the Moore-Penrose generalized inverse of $A$. $\mathscr{R}(A)$ denotes the range of $A$. The $m \times m$ unit matrix will be denoted by $I_{m}$ or by $I$, and its $i$ th column by $e_{i}$, while $e \equiv(1,1, \ldots, 1)^{\mathrm{T}}$. Throughout we use the norm $\|x\|=\|x\|_{2} \equiv \sqrt{x^{\mathrm{T}} x}$ for vectors, and if a matrix $A$ has singular values $\sigma_{1} \geqslant \cdots \geqslant \sigma_{n} \geqslant 0$, we write $\|A\|=\|A\|_{2}=\sigma_{1}$. We say $A$ is ill-conditioned if $\sigma_{1} / \sigma_{n}$ is very large. We will use $\mathscr{E}\{\cdot\}$ to denote the expected value, and $\operatorname{cov}\{\cdot\}$ to denote the covariance, that is $\operatorname{cov}\{x\}=\mathscr{E}\left\{(x-\mathscr{E}\{x\})(x-\mathscr{E}\{x\})^{\mathrm{T}}\right\}$. The notation $u \sim \mathrm{N}(\bar{u}, U)$ will mean $u$ is a normally distributed random vector with mean $\bar{u}$ and covariance $U$.

\section{The linear model and some background}

We will assume that at a given time we have a general linear model with positive definite noise covariance matrix

$$
y^{G}=G^{G} z+v^{G}, \quad v^{G} \sim \mathrm{N}\left(0, \sigma^{2} L L^{\mathrm{T}}\right),
$$


where $L$ is lower triangular with positive diagonal. Defining $y \equiv L^{-1} y^{G}, G \equiv L^{-1} G^{G}$, $v \equiv L^{-1} v^{G}$ gives the standard linear model

$$
y=G z+v, \quad v \sim \mathrm{N}\left(0, \sigma^{2} I\right) .
$$

The model will vary with time. In GPS, time is discretized into points, called epochs, and the time period between two consecutive epochs depends on the sampling rate at which a GPS receiver collects and stores the measurements.

In GPS, two main sets of measurements are used: one set is called "code" measurements (or "pseudorange" measurements), and the other "carrier phase" measurements, see for example Hofmann-Wellenhof et al. (1997). We often have

$$
z \equiv\left[\begin{array}{l}
x \\
a
\end{array}\right]
$$

where $x$ denotes the position to be estimated (from some known position), and $a$ is either some element, such as a receiver clock bias in the code-based problem, or some vector, as is the case for the carrier phase based problem (see for example Hofmann-Wellenhof et al., 1997, Section 9.4; Chang et al., 2000). In our general model we will assume $G^{G}$, and so $G$, is $m \times n$ where there can be a fault (failure, outlier) in any one of the first $m_{s} \leqslant m$ observations, but not in the last $m-m_{s}$ observations. In the code-based problem we will usually have $m=m_{s}$ (so we will not distinguish between $m$ and $m_{s}$ ), but in the carrier phase-based problem we usually have $m>m_{s}$. For example, the first $m_{s}$ elements of $y^{G}$ could be measurements from the $m_{s}$ satellites, while the remaining elements of $y^{G}$ could be estimates from other computations. In order for the deletion of the $i$ th equation, $1 \leqslant i \leqslant m_{s}$, to fully eliminate any fault in the $i$ th measurement (i.e. from the $i$ th satellite in the GPS case), we will require that

$$
L L^{\mathrm{T}}=\left[\begin{array}{cc}
D^{2} & 0 \\
0 & H^{(22)}
\end{array}\right], \quad \text { so } L=\left[\begin{array}{cc}
D & 0 \\
0 & L^{(22)}
\end{array}\right], \quad D \text { (positive) diagonal. }
$$

If the noise components of the satellite signals are uncorrelated, then the equations can usually be developed so that the covariance has this form. Then any fault in the $i$ th measurement, $1 \leqslant i \leqslant m_{s}$, of our general model becomes a fault in the $i$ th measurement alone of the standard model. Because we will now focus on the standard linear model (2) with a possible fault, the exposition will lose nothing by assuming $m_{s}=m$ for simplicity.

Our linear model with possible faults is then

$$
y=G z+v+b, \quad v \sim \mathrm{N}\left(0, \sigma^{2} I\right),
$$

where $G$ is an $m \times n$ matrix, $m \geqslant n$, noise $v$ is normally distributed, and $b$ satisfies

$$
b= \begin{cases}e_{i} \beta & \text { for some } \beta \neq 0 \text { if there is a fault in measurement } i, 1 \leqslant i \leqslant m, \\ 0 & \text { if there is no fault. }\end{cases}
$$


This assumes there is at most one fault. In all aspects of integrity we assume reliability is such that we need only ever deal with the possibility of a fault in one input. We cannot know a priori which input, or if there is a fault at all, but this assumption makes the problem tractable. Also, it is extremely unlikely that more than one GPS satellite has a fault at any given time.

When $G$ is rank deficient we do not have a unique estimate. Otherwise, let full column rank $G$ have the $Q R$ factorization, with orthogonal $Q$ and upper triangular $R$

$$
G=\left[Q_{1}, Q_{2}\right]\left[\begin{array}{l}
R \\
0
\end{array}\right]=Q\left[\begin{array}{l}
R \\
0
\end{array}\right], \quad m \times n Q_{1}, \quad m \times(m-n) Q_{2} .
$$

$Q_{2}$ is arbitrary up to an orthogonal right multiplicative factor $U$, as in $Q_{2} U$. The least squares (LS) estimator of $z$ in (4) is

$$
\begin{aligned}
& \hat{z} \equiv G^{\dagger} y=R^{-1} Q_{1}^{\mathrm{T}} y=z+R^{-1} Q_{1}^{\mathrm{T}}(v+b), \\
& \mathscr{E}\{\hat{z}-z\}=R^{-1} Q_{1}^{\mathrm{T}} b, \quad \operatorname{cov}\{\hat{z}-z\}=\sigma^{2}\left(R^{\mathrm{T}} R\right)^{-1}=\sigma^{2}\left(G^{\mathrm{T}} G\right)^{-1},
\end{aligned}
$$

which is also the best linear unbiased estimator (BLUE) for (4) when $b=0$. When $b=0$, the error covariance matrix $\operatorname{cov}\{\hat{z}-z\}$ defines an ellipsoid with center 0 in which $\hat{z}-z$ lies with a certain probability. For general discussion, we will use the ellipsoid whose semi-axis lengths are the singular values of $\sigma R^{-1}$. Note $\left\|\sigma^{2}\left(G^{\mathrm{T}} G\right)^{-1}\right\|_{2}$ is the square of the largest semi-axis length.

We will be interested in adding and deleting rows of $G$ (for example when we add or delete satellite measurements). Let $n \times m G^{\mathrm{T}} \equiv\left[g, \bar{G}^{\mathrm{T}}\right]$, and $\lambda_{1}(\cdot) \geqslant \cdots \geqslant \lambda_{n}(\cdot)$ and $\sigma_{1}(\cdot) \geqslant \cdots \geqslant \sigma_{n}(\cdot)$ denote real eigenvalues and singular values, respectively, then $G^{\mathrm{T}} G=\bar{G}^{\mathrm{T}} \bar{G}+g g^{\mathrm{T}}$, so from standard eigenvalue perturbation results (Golub and Van Loan, 1996, Section 8.1.1) we know that for $i=1, \ldots, n$

$$
\begin{aligned}
& \lambda_{i}\left(\bar{G}^{\mathrm{T}} \bar{G}\right)=\sigma_{i}^{2}(\bar{G}) \leqslant \lambda_{i}\left(G^{\mathrm{T}} G\right)=\sigma_{i}^{2}(G) \leqslant \lambda_{i}\left(\bar{G}^{\mathrm{T}} \bar{G}\right)+\|g\|_{2}^{2}=\sigma_{i}^{2}(\bar{G})+\|g\|_{2}^{2}, \\
& \sigma^{2}\left\|\left(\bar{G}^{\mathrm{T}} \bar{G}\right)^{-1}\right\|_{2}=\frac{\sigma^{2}}{\sigma_{n}^{2}(\bar{G})} \geqslant \sigma^{2}\left\|\left(G^{\mathrm{T}} G\right)^{-1}\right\|_{2}=\frac{\sigma^{2}}{\sigma_{n}^{2}(G)},
\end{aligned}
$$

and no semi-axis can be made larger by adding a row to $G$, or smaller by deleting a row. We will show in Section 3.4 how deleting some rows can lead to very large semi-axes.

Whether $\left(G^{\mathrm{T}} G\right)^{-1}$ is small or large, if (4) was an accurate model and we knew $b=0$, then this error covariance matrix would allow us to give an ellipsoid in which we knew $(\hat{z}-z) / \sigma$ must lie with probability 0.999 . If

$$
z \equiv\left[\begin{array}{l}
x \\
a
\end{array}\right]
$$

where $x$ denotes the position to be estimated, and

$$
\hat{z} \equiv\left[\begin{array}{l}
\hat{x} \\
\hat{a}
\end{array}\right]
$$


we could obtain "radials" from this - the horizontal radial describing a distance from $\hat{x}$ within which the horizontal component of $x$ must lie with probability at least 0.999 . Thus, horizontal radials describe circular disks in the horizontal plane. Similarly, we could obtain a $99.9 \%$ vertical radial, essentially describing a vertical disk. Clearly the horizontal and vertical radials usually have different values. But in GPS we cannot know a priori that $b=0$, and integrity algorithms are needed to give us the same sort of security in the presence of a possible nonzero but unknown $b$ of the form (5). The actual radials must include this possibility.

We will not show how to obtain radials here, but will briefly refer to the resulting region in which we are sure the aircraft lies (with a probability of at least 0.999, even in the presence of a nonzero $b$ ) as the "envelope". Instead of obtaining radials we will focus on some more fundamental scalars $\omega_{i i} \in[0,1]$. These are easy to compute and understand, and it can be shown that small $\omega_{i i}$ lead to large radials (the radials depend directly on the $\omega_{i i}^{-1}$ ). But the theoretical importance of the $\omega_{i i}$ is broader than this, and their fundamental and pervasive nature needs to be more widely recognized in the GPS integrity area, although we realize that they have been mentioned in the estimation literature (Cook and Weisberg, 1982, Section 2.1). For example, they can be used to compute the test statistics, and the sensitivities of these statistics depend greatly on them. Also they provide a direct understanding as to why the envelope will be large (the position will be very uncertain) when an $\omega_{i i}$ is small. They appear as key components in the numerical linear algebra theory and computations we develop, and they help us to understand the effects of modeling and rounding errors.

"Geometry" in the GPS literature refers to the relative positions of the satellites and receivers. There are two major ways in which geometry can adversely affect GPS calculations, and we discuss the first here. The second will be discussed later. The geometry is poor for estimating $z$ in (4) if $(\hat{z}-z) / \sigma$ has large covariance, see Kelly (1998), so that $\hat{z}$ can be a poor estimator of $z$ even when $b=0$. In this example, large $\left(G^{\mathrm{T}} G\right)^{-1}$ corresponds to some of the columns of $G$ not defining $\mathscr{R}(G)$ well. Since the satellites are well separated, having measurements from more satellites (more rows of $G$ ) would usually significantly reduce the error covariance for the present case. Note that the matrix $G$ essentially summarizes this geometry.

When $m>n$ the parity vector for the model (4) is defined to be (since $Q_{2}^{\mathrm{T}} G=0$ )

$$
p \equiv Q_{2}^{\mathrm{T}} y=Q_{2}^{\mathrm{T}}(v+b), \quad \mathscr{E}\{p\}=Q_{2}^{\mathrm{T}} b, \quad \operatorname{cov}\{p\}=\sigma^{2} I_{m-n} .
$$

This is arbitrary up to orthogonal $U$ in $U^{\mathrm{T}} p$, where this is the same $U$ as in the comment following (6), but $\left\|U^{\mathrm{T}} p\right\|_{2}=\|p\|_{2}$ is not arbitrary. With the least squares estimator (7), the LS residual $r$ is not in any way arbitrary:

$$
\begin{aligned}
& r \equiv y-G \hat{z}=Q_{2} Q_{2}^{\mathrm{T}} y=Q_{2} p=Q_{2} Q_{2}^{\mathrm{T}}(v+b), \\
& \mathscr{E}\{r\}=Q_{2} Q_{2}^{\mathrm{T}} b, \quad \operatorname{cov}\{r\}=\sigma^{2} Q_{2} Q_{2}^{\mathrm{T}} .
\end{aligned}
$$


Here we define the $\omega_{i i}\left(r\right.$ and $\omega_{i i}$ are unaltered by $U$, and $\rho_{i}$ is the $i$ th element of $r$ )

$$
\begin{aligned}
& \omega_{i i}^{2} \equiv e_{i}^{\mathrm{T}} Q_{2} Q_{2}^{\mathrm{T}} e_{i}, \quad 1 \geqslant \omega_{i i} \geqslant 0, \quad \sum_{i=1}^{m} \omega_{i i}^{2}=\operatorname{trace}\left(Q_{2} Q_{2}^{\mathrm{T}}\right)=m-n \geqslant 0, \\
& \omega_{i i}=0 \Leftrightarrow e_{i}^{\mathrm{T}} Q_{2}=0 \Rightarrow\left\{\rho_{i} \equiv e_{i}^{\mathrm{T}} r=0 \text { and } \mathscr{E}\left\{\rho_{i}\right\}=0 \text { and } \operatorname{cov}\left\{\rho_{i}\right\}=0\right\} .
\end{aligned}
$$

The quantities in $(8)-(10)$ are used to detect and isolate faults in the input data. In particular Brenner (1990) makes careful use of $Q_{2}$ in $(6) . \mathscr{R}\left(Q_{2}^{\mathrm{T}}\right)$ is sometimes referred to as the parity space, since this is the space in which the parity vector $p=Q_{2}^{\mathrm{T}} y$ in (8) must lie. Note that $\mathscr{R}\left(Q_{2}\right)$ could be called the residual space, since the residual is always in this space, see (9), and $Q_{2} Q_{2}^{\mathrm{T}}$ is the orthogonal projector onto this space.

The case of $\omega_{i i}=0$ is in theory possible, because it is trivial to construct examples of general $G$ for which $e_{i}^{\mathrm{T}} Q_{2}=0$. Note from (10) there can be at most $n$ such $i$. The possibility that $\omega_{i i} \equiv\left\|Q_{2}^{\mathrm{T}} e_{i}\right\|_{2}=0$ is interesting because if we also have a fault in the $i$ th measurement, so $b=e_{i} \beta$, then $Q_{2}^{\mathrm{T}} b=0$, and no trace of $b$ will appear in $p$ or $r$ in (8) or (9), so the fault could not be correctly detected by using just these two vectors.

Similarly, small $\omega_{i i}$ will mean a fault of this form is unlikely to be detected with a simple test. This is an example of our second, and the more difficult form of bad geometry: geometry that obscures possible faults. We discuss some basic aspects of this below, and show in Section 3 that a small $\omega_{i i}$ leads to a large error covariance matrix when the $i$ th observation is deleted. It can be shown that small $\omega_{i i}$ also lead to large radials. This is logical-if you can hardly detect something that could be causing a large error, then your error bounds must be that much larger to take this into account. Thus, small $\omega_{i i}$ and large radials are two (closely related) indicators of the same problem. Here, we concentrate on the $\omega_{i i}$. From the above discussion, we see the scalars $\omega_{i i}$ could be called the "fault observability scalars", since $\omega_{i i}$ determines how easily a fault $e_{i} \beta$ can be detected. Before going more deeply into the mathematics, we will describe some obvious effects these $\omega_{i i}$ have.

The $Q R$ factorization (6) divides the space of observations into $\mathscr{R}\left(Q_{1}\right)$ and $\mathscr{R}\left(Q_{2}\right)$. Let $y=y_{1}+y_{2}, y_{1} \equiv Q_{1} Q_{1}^{\mathrm{T}} y \in \mathscr{R}\left(Q_{1}\right), y_{2} \equiv Q_{2} Q_{2}^{\mathrm{T}} y \in \mathscr{R}\left(Q_{2}\right)$, then the LS estimator satisfies the compatible system $G \hat{z}=y_{1}$, with LS residual $r \equiv y-G \hat{z}=y_{2}$. Suppose this corresponds to no fault, and now we add a fault $b$. Let $b=b_{1}+b_{2}, b_{1} \equiv Q_{1} Q_{1}^{\mathrm{T}} b \in \mathscr{R}\left(Q_{1}\right)$, $b_{2} \equiv Q_{2} Q_{2}^{\mathrm{T}} b \in \mathscr{R}\left(Q_{2}\right)$. The part $b_{1}$ of $b$ only alters the LS estimator $\hat{z}$ to the solution of the compatible system $G(\hat{z}+\delta \hat{z})=y_{1}+b_{1}$, so $G \delta \hat{z}=b_{1}$, while $b_{2}$ only changes the residual to $y_{2}+b_{2}$.

First, let us consider the (very unlikely) extremes. If $b \in \mathscr{R}\left(Q_{2}\right)$ it does not alter $\hat{z}$, but all of it changes the residual. On the other hand, if $b \in \mathscr{R}\left(Q_{1}\right)$ (so $b \perp \mathscr{R}\left(Q_{2}\right)$ ), $b$ only alters $\hat{z}$ and cannot be seen in the residual! Remember that $0 \neq b=e_{i} \beta \perp \mathscr{R}\left(Q_{2}\right)$ means $\omega_{i i}=\left\|Q_{2}^{\mathrm{T}} e_{i}\right\|=0$, so $\omega_{i i}=0$ means we cannot detect a fault $b=e_{i} \beta$, and all the effect of any such fault would appear in the estimator $\hat{z}$. Since we cannot know if there is such a fault, and if there is, how large it is, we cannot include this $i$ th observation and still have integrity. 
Thus, if $\omega_{i i}=0$ the $i$ th observation must be discarded, which we will show in Section 3.2 has serious consequences. Since $\omega_{i i}=0 \Rightarrow \rho_{i}=0$, see (10), this presents the apparent contradiction of discarding an observation for which the LS estimate gives zero residual. But it is the geometry (the form of $Q_{2}$, and so of $G$ ) that led to $\rho_{i}=0$ necessarily-independently of the observation, so this is not contradictory. If $\tau$ is a good upper bound on the relative accuracy of $G$ and $\omega_{i i} \leqslant \tau$, then we will show in Section 3.3 that the true $\omega_{i i}$ could be zero, and again the $i$ th observation should be dropped.

The other extreme for $\omega_{i i}$ corresponds to $\omega_{i i}^{2} \equiv e_{i}^{\mathrm{T}} Q_{2} Q_{2}^{\mathrm{T}} e_{i}=1$. But $I=Q_{1} Q_{1}^{\mathrm{T}}+Q_{2} Q_{2}^{\mathrm{T}}$, so this is equivalent to $e_{i}^{\mathrm{T}} Q_{1} Q_{1}^{\mathrm{T}} e_{i}=0$, that is the $i$ th row of $Q_{1}$ is zero. Since $G=Q_{1} R$ this is equivalent to the $i$ th row of $G$ being zero; also it means the $i$ th observation makes no contribution to $\hat{z}$, see (7). This means if $\omega_{i i}=1$ there is no need to drop the $i$ th observation even if it is clearly faulty. However, there is no cost in dropping it since the $Q R$ factorization will be unchanged, and since in GPS a fault in one epoch suggests that that particular input is unreliable, it should be dropped there. Of course, a model with a zero row in $G$ would be a strange one. Between these two easily handled extremes $-\omega_{i i}=0$ where any fault could not be detected but could cause great harm, and $\omega_{i i}=1$ where a fault would almost certainly be detected but would cause no harm-lie almost all cases: $0<\omega_{i i}<1$.

\section{Reduced models}

If we eliminate one or more observations (rows) from our linear model (4), we will refer to the resulting model as a reduced model. In Section 4 we will show how these reduced models lead to important statistics, but we first develop the basic theory here.

The elements $\omega_{i i}$ in (10) play a key role in the study of integrity. In this section we will also show that when one is very small, the geometry does not support integrity, while if one is fairly small, we cannot delete the corresponding measurement and still have an acceptable envelope.

We will first describe a good method for updating the $Q R$ factorization when a row is removed (sometimes called "downdating") and all of $Q$ is available, whether $\omega_{i i}$ is small or not. This is useful in GPS, but it will also allow us to understand the drastic consequences of deleting the $i$ th row of $G$ when $\omega_{i i}=0$, and why integrity cannot then be supported. Then we will look at the effect of possible errors in the model on this decision. Finally, we will show the bad effect small $\omega_{i i}$ has on the error covariance of the reduced model with $i$ th observation removed.

\subsection{An algorithm for deleting an observation}

Suppose we have $Q=\left[Q_{1}, Q_{2}\right]$ and $R$ in the $Q R$ factorization $G=Q_{1} R$ of $m \times n G$ with rank $n$. Without loss of generality we wish to find the $Q R$ factorization of $G$ less its first row $g^{\mathrm{T}}$ say. We can do this by introducing products of the form $I_{m}=J_{i j} J_{i j}^{\mathrm{T}}$ 
between $Q$ and

$$
\left[\begin{array}{l}
R \\
0
\end{array}\right],
$$

where $J_{i j}$ is a rotation in the $\{i, j\}$-plane (see, for example, Golub and Van Loan, 1996, Section 12.5.3). We choose a sequence designed to zero the $m$ th, then $(m-1)$ st, down to the second element of the first row of $Q$. For illustration let $m=4$ and $n=2$. In the first step we choose $J_{3,4}$ so that $Q J_{3,4}$ is zero in position $(1,4)$ :

$$
\begin{aligned}
G & =\left[\begin{array}{c}
g^{\mathrm{T}} \\
\bar{G}
\end{array}\right]=Q\left[\begin{array}{l}
R \\
0
\end{array}\right]=\left[\begin{array}{llll}
q_{1} & q_{2} & q_{3} & q_{4}
\end{array}\right] J_{3,4} J_{3,4}^{\mathrm{T}}\left[\begin{array}{cc}
\times & \times \\
0 & \times \\
0 & 0 \\
0 & 0
\end{array}\right] \\
& =f\left[q_{1}\left|q_{2}\right| \tilde{q}_{3} \mid \begin{array}{c}
\tilde{q}_{4} \\
\tilde{q}_{4}
\end{array}\right]\left[\begin{array}{cc}
\rho_{11} & \rho_{12} \\
0 & \rho_{22} \\
0 & 0 \\
0 & 0
\end{array}\right] .
\end{aligned}
$$

Since $m \geqslant n+2$, this first rotation did not alter $R$. We assume we choose rotations so each $\zeta_{j} \geqslant 0$ here. Note, in general, the rotations $J_{m-1, m}, J_{m-2, m-1}, \ldots, J_{n+1, n+2}$ lead to $\zeta_{n+1}=\left\|e_{1}^{\mathrm{T}} Q_{2}\right\|_{2}=\omega_{11}$. We continue with $J_{2,3}$ and finally $J_{1,2}$ in our small example:

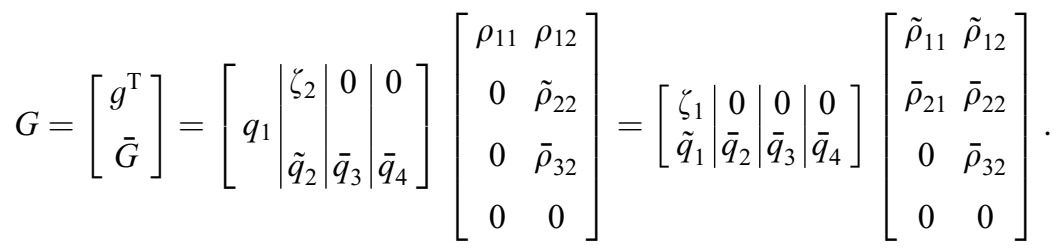

But the first row of $Q J_{3,4} J_{2,3} J_{1,2}$ has length unity, so $\zeta_{1}=1$. Also this row is orthogonal to every other row, so $\tilde{q}_{1}=0$. Finally, $\bar{q}_{2}, \bar{q}_{3}, \bar{q}_{4}$ are orthonormal, so the $Q R$ factorization of $\bar{G}$ is

$$
\bar{G}=\left[\begin{array}{lll}
\bar{q}_{2} & \bar{q}_{3} & \bar{q}_{4}
\end{array}\right]\left[\begin{array}{cc}
\bar{\rho}_{21} & \bar{\rho}_{22} \\
0 & \bar{\rho}_{32} \\
0 & 0
\end{array}\right]=\bar{Q}\left[\begin{array}{l}
\bar{R} \\
0
\end{array}\right] .
$$

If $\omega_{11} \neq 0$ here, it can be seen from the sequence of rotations that $\bar{R}$ is $n \times n$ and nonsingular, so $\bar{G}$ has rank $n$.

It is obvious how this applies to general $m$ and $n$, and how it can be continued to delete further rows. It is computationally fast for significant $m$ and $n$, costing $O(m n)$ floating point operations, and is numerically reliable. But an additional advantage is that it shows what happens when an $\omega_{11}=0$. 


\subsection{The effect of a zero $\omega_{i i}$}

If $\omega_{11}=0$ then the first row of $Q_{2}$ is zero, so the $J_{i, j}$ above are only applied to $Q_{1}$, and $J_{i, j}^{\mathrm{T}}$ are applied only to $R$, giving after these transformations

$$
G=\left[\begin{array}{c}
g^{\mathrm{T}} \\
\bar{G}
\end{array}\right]=\left[Q_{1} \mid Q_{2}\right]\left[\begin{array}{c}
R \\
\overline{0}
\end{array}\right]=\left[\begin{array}{cc|c}
1 & 0 & 0 \\
0 & \bar{Q}_{1} & \bar{Q}_{2}
\end{array}\right]\left[\begin{array}{c}
g^{\mathrm{T}} \\
\bar{R} \\
\overline{0}
\end{array}\right]=\left[\begin{array}{c}
g^{\mathrm{T}} \\
\overline{Q_{1}} \bar{R}
\end{array}\right] .
$$

This means that when the first row is deleted, $\bar{R}$ above has only $n-1$ nonzero rows. In our example this corresponds to the element $\bar{\rho}_{32}$ being zero. Since $\bar{R}$ has rank $n-1$, $\bar{G}$ must necessarily have rank $n-1$. But this means any estimator found using $\bar{G}$ is not uniquely determined! It corresponds to the covariance of the estimate not being defined, see (7).

We write the finding above as a theorem.

Theorem 3.1. Let the $m \times n$ matrix $G$ have full column rank. Let $Q_{2}$ have orthonormal columns and $\mathscr{R}\left(Q_{2}\right)=\mathscr{R}^{\perp}(G)$. Denote the remaining matrix of $G$ by $\bar{G}$ after the ith row is deleted. Then $\omega_{i i} \equiv\left\|Q_{2}^{\mathrm{T}} e_{i}\right\|_{2}=0$ if and only if $\operatorname{rank}(\bar{G})=n-1$.

This exercise has several interesting lessons. First, if in some problem $\omega_{i i}=0$ occurred, it would mean that using the $i$ th observation would lead to a loss of integritysee our earlier discussions. But deleting the $i$ th observation would lead to a loss of uniqueness in the estimate, and so to a loss of integrity. Thus $\omega_{i i}=0$ means the geometry cannot support integrity. In GPS code-based tracking where each observation corresponds to a satellite, this means deleting the $i$ th observation would lead to the remaining $m-1$ satellites giving a geometry where the $(m-1) \times n \bar{G}$ had $\operatorname{rank} n-1$. This would be highly unlikely unless $m=n$ already.

Here we would like to make some remarks on Theorem 3.1. In fact Theorem 3.1 can also be derived from a result of Stewart (1979), which is concerned with the Cholesky downdating problem: given a nonsingular $n \times n$ upper triangular matrix $R$ and an $n$-vector $g$, find the Cholesky factor $\bar{R}$ of $R^{\mathrm{T}} R-g g^{\mathrm{T}}$, i.e., $\bar{R}^{\mathrm{T}} \bar{R}=R^{\mathrm{T}} R-g g^{\mathrm{T}}$. By the algebraic operation

$$
R^{\mathrm{T}} R-g g^{\mathrm{T}}=R^{\mathrm{T}}\left(I-R^{-\mathrm{T}} g g^{\mathrm{T}} R^{-1}\right) R .
$$

Stewart showed that $R^{\mathrm{T}} R-g g^{\mathrm{T}}$ is positive definite if and only if $\left\|R^{-\mathrm{T}} g\right\|<1$. From his proof, we can observe that $R^{\mathrm{T}} R-g g^{\mathrm{T}}$ is singular if and only if $\left\|R^{-\mathrm{T}} g\right\|=1$. If as before $R$ denotes the $R$-factor of the $Q R$ factorization of our matrix $G, g^{\mathrm{T}}$ the $i$ th row of $G$, and $\bar{G}$ the remaining matrix of $G$ after $g^{T}$ is deleted, then

$$
\bar{G}^{\mathrm{T}} \bar{G}=G^{\mathrm{T}} G-g g^{\mathrm{T}}=R^{\mathrm{T}} R-g g^{\mathrm{T}} .
$$

Since $g=G^{\mathrm{T}} e_{i}$ and $G=Q_{1} R$,

$$
\left\|R^{-\mathrm{T}} g\right\|^{2}=\left\|R^{-\mathrm{T}} G^{\mathrm{T}} e_{i}\right\|^{2}=\left\|Q_{1}^{\mathrm{T}} e_{i}\right\|^{2}=1-\left\|Q_{2}^{\mathrm{T}} e_{i}\right\|^{2}=1-\omega_{i i}^{2} .
$$


Thus by the above result of Stewart, $\bar{G}$ is singular (so $\operatorname{rank}(\bar{G})=n-1$ ) if and only if $\omega_{i i}=0$.

From (12) it was also shown in Stewart (1979) that for the smallest singular value of $\bar{R}$ we have the following upper bound:

$$
\sigma_{\min }(\bar{R}) \leqslant\|R\| \sqrt{1-\left\|R^{-\mathrm{T}} g\right\|^{2}} .
$$

Thus Stewart observed that if $\left\|R^{-\mathrm{T}} g\right\|$ is near unity, i.e., $\omega_{i i}$ is near zero in our case, $\sigma_{\min }(\bar{R})$, which is equal to $\sigma_{\min }(\bar{G})$ in our case, will be small. Later in Section 3.4 we use our approach to derive a better bound on $\sigma_{\min }(\bar{G})$ and discuss the effects of a small $\omega_{i i}$ on the error covariance of the reduced model.

\subsection{The case of a very small $\omega_{i i}$}

A more likely case is that of very small $\omega_{i i}$. We have remarkably accurate linear models in GPS, but they are not perfect. Let $\Delta G$ denote the difference between the ideal $\hat{G}=G+\Delta G$ and the $G$ we are computing with. Suppose the best relative bound we have on such deviations is $\tau$ (we assume $\tau<1$ ), so that we know

$$
\|\Delta G\|_{2} \leqslant \tau\|G\|_{2} .
$$

(The theory is almost identical if we use the Frobenius norm). We can assume (15) also includes the effect of finite precision computation when we use numerically stable algorithms. Thus, even if $G$ is known exactly, our answers will only be true for some $G+\Delta G$ where $\tau$ in (15) is $O(\varepsilon), \varepsilon$ being the floating point precision of the computer. We will show the following.

Theorem 3.2. Let (15) describe the accuracy of our linear model. Whenever we have $a$ with

$$
\omega_{i i} \leqslant \tau, \quad \text { for some } i=1, \ldots, m,
$$

we can find $\hat{G}=G+\Delta G$ with $\hat{\omega}_{i i}=0$ and $\Delta G$ satisfying (15).

Proof. Without loss of generality we can consider $i=1$. Since $Q_{2}$ is arbitrary up to orthogonal $U$ in $Q_{2} U$ we can choose $U_{1}$ so that $e_{1}^{\mathrm{T}} Q_{2} U_{1}=\omega_{11} e_{1}^{\mathrm{T}}$. Now apply orthogonal $J$ in $G=Q_{1} J J^{\mathrm{T}} R \equiv \tilde{Q}_{1} J^{\mathrm{T}} R$ so $e_{1}^{\mathrm{T}} \tilde{Q}_{1}=\gamma_{11} e_{n}^{\mathrm{T}}>0$. It follows from this that

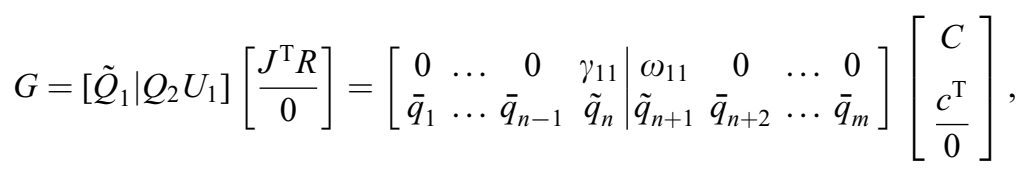

where

$$
\left[\begin{array}{c}
C \\
c^{\mathrm{T}}
\end{array}\right] \equiv J^{\mathrm{T}} R
$$


and since the first row of $Q$ has length unity, $\gamma_{11}^{2}+\omega_{11}^{2}=1$. With the notation in Section 3.1, taking

$$
J=J_{n-1, n} J_{n-2, n-1} \cdots J_{1,2}\left[e_{2}, e_{3}, \ldots, e_{n}, e_{1}\right]
$$

leaves $C$ upper trapezoidal. Note that $\gamma_{11}$ and $\omega_{11}$ form the core of a useful $m \times m$ rotation matrix $J_{n, n+1}=\operatorname{diag}\left(I_{n-1}, \tilde{J}, I_{m-n-1}\right)$ where

$$
\tilde{J} \equiv\left[\begin{array}{cc}
\gamma_{11} & -\omega_{11} \\
\omega_{11} & \gamma_{11}
\end{array}\right], \quad\left[\begin{array}{cc}
\gamma_{11} & \omega_{11} \\
\tilde{q}_{n} & \tilde{q}_{n+1}
\end{array}\right] \tilde{J}=\left[\begin{array}{ll}
1 & 0 \\
0 & \bar{q}
\end{array}\right], \quad \tilde{J}^{\mathrm{T}}\left[\begin{array}{c}
c^{\mathrm{T}} \\
0
\end{array}\right]=\left[\begin{array}{c}
\gamma_{11} c^{\mathrm{T}} \\
-\omega_{11} c^{\mathrm{T}}
\end{array}\right] .
$$

Applying $J_{n, n+1} J_{n, n+1}^{\mathrm{T}}$ between the two factors of $G$ in (17) gives

$$
\begin{aligned}
{\left[\begin{array}{c}
g^{\mathrm{T}} \\
\bar{G}
\end{array}\right] } & =G=\left[\begin{array}{cccc|cccc}
0 & \ldots & 0 & 1 & 0 & 0 & \ldots & 0 \\
\bar{q}_{1} & \ldots & \bar{q}_{n-1} & 0 & \bar{q} & \bar{q}_{n+2} & \ldots & \bar{q}_{m}
\end{array}\right]\left[\begin{array}{c}
C \\
\frac{\gamma_{11} c^{\mathrm{T}}}{-\omega_{11} c^{\mathrm{T}}} \\
0
\end{array}\right] \\
& \equiv\left[\hat{Q}_{1} \mid \hat{Q}_{2}\right]\left[\begin{array}{c}
C \\
\frac{\gamma_{11} c^{\mathrm{T}}}{-\omega_{11} c^{\mathrm{T}}} \\
0
\end{array}\right] .
\end{aligned}
$$

This shows us that $g^{\mathrm{T}}=\gamma_{11} c^{\mathrm{T}}$ and

$$
\begin{aligned}
& \hat{G}=G+\Delta G \equiv G+\left[\begin{array}{l}
0 \\
\bar{q}
\end{array}\right] \omega_{11} c^{\mathrm{T}}=\left[\hat{Q}_{1} \mid \hat{Q}_{2}\right]\left[\begin{array}{c}
C \\
\frac{\gamma_{11} c^{\mathrm{T}}}{0}
\end{array}\right] \equiv\left[\hat{Q}_{1} \mid \hat{Q}_{2}\right]\left[\frac{\hat{C}}{0}\right], \\
& \|\Delta G\|_{2}=\omega_{11}\|c\|_{2}=\frac{\omega_{11}\|g\|_{2}}{\sqrt{1-\omega_{11}^{2}}} \leqslant \omega_{11}\|R\|_{2}=\omega_{11}\|G\|_{2},
\end{aligned}
$$

see (17), so $\Delta G$ satisfies (15) when (16) holds, and $\hat{Q}_{2}$ has zero first row. Matrix $\hat{C}$ is $n \times n$ nonsingular, and by applying an orthogonal matrix to its left to bring it to upper triangular, and the transpose of the same orthogonal matrix to the right of $\hat{Q}_{1}$, gives us the $Q R$ factorization of $\hat{G}$ with $\hat{Q}_{2}$ unchanged, so $\hat{\omega}_{11}=0$.

If (16) is satisfied, then the above theorem tells us that the $G$ we are computing with could have come from an ideal $\hat{G}$ which did not support integrity, and again we have to assume our present geometry cannot support integrity.

We have seen that under the choice of $\Delta G$ in (19) $\hat{G}=G+\Delta G$ has $\hat{\omega}_{11}=0$. An interesting theoretical problem is to find $\Delta G$ which has the minimum 2-norm (or Frobenius norm) among all $\Delta G$ which make $\hat{\omega}_{11}=0$. We have the following result.

Theorem 3.3. Let $G \in \mathscr{R}^{m \times n}$ have full column rank. Let $g^{\mathrm{T}}$ be the ith row of $G$ and let $\bar{G}$ be the remaining matrix of $G$ after $g^{T}$ is deleted. If $\sigma_{\min }(G)>\sigma_{\min }(\bar{G})$, then

$$
\min _{\hat{\omega}_{i i}=0, \operatorname{rank}(\hat{G})=n}\|\hat{G}-G\|_{2}=\sigma_{\min }(\bar{G}) \leqslant \frac{\omega_{i i}\|g\|_{2}}{\sqrt{1-\omega_{i i}^{2}}} .
$$


Proof. Without loss of generality, we assume $i=1$. Let

$$
\Delta G \equiv\left[\begin{array}{c}
\Delta g^{\mathrm{T}} \\
\Delta \bar{G}
\end{array}\right] \quad \text { so } \hat{G}=G+\Delta G=\left[\begin{array}{c}
g^{\mathrm{T}}+\Delta g^{\mathrm{T}} \\
\bar{G}+\Delta \bar{G}
\end{array}\right] \text {. }
$$

From Theorem 3.1, if $\hat{G}$ has rank $n$, then $\hat{w}_{11}=0$ if and only if $\bar{G}+\Delta \bar{G}$ has rank $n-1$. From the singular value theory (see, for example, Theorem 2.5.3 in Golub and Van Loan, 1996), the following $\Delta \bar{G}_{0}$ has the minimum 2-norm (or Frobenius norm) among all matrices $\Delta \bar{G}$ which make the rank of $\bar{G}+\Delta \bar{G}$ equal to $n-1$ :

$$
\Delta \bar{G}_{0}=-\bar{\sigma}_{n} \bar{u}_{n} \bar{v}_{n}^{\mathrm{T}}
$$

where $\bar{\sigma}_{n}$ is the smallest singular value of $\bar{G}, \sigma_{\min }(\bar{G})$, and $\bar{u}_{n}$ and $\bar{v}_{n}$ are the corresponding left and right singular vectors. Take

$$
\Delta G_{0}=\left[\begin{array}{c}
0 \\
-\bar{\sigma}_{n} \bar{u}_{n} \bar{v}_{n}^{\mathrm{T}}
\end{array}\right] .
$$

Since $\left\|\Delta G_{0}\right\|_{2}=\sigma_{\min }(\bar{G})<\sigma_{\min }(G), \hat{G}$ must have rank $n$. Thus, $\Delta G_{0}$ has the minimum 2-norm (or Frobenius norm) making $\hat{\omega}_{11}=0$ corresponding to $\hat{G}_{0}=G+\Delta G_{0}$. Since (20) holds for the special $\Delta G$ in (19), we must have that

$$
\left\|\Delta G_{0}\right\|_{2} \leqslant \frac{\omega_{11}\|g\|_{2}}{\sqrt{1-\omega_{11}^{2}}}
$$

which will also be shown directly in the next section.

\subsection{The error covariance of the reduced model}

We now show that if $\omega_{i i}$ is small, then deleting the $i$ th observation results in the reduced model having a large error covariance. This lesson also comes from (18). We see that $g^{\mathrm{T}}=\gamma_{11} c^{\mathrm{T}}$, and $\hat{Q}_{1} e_{n}=e_{1}$, and deleting the first row of $G$ gives orthogonal $\left[\bar{Q}_{1} \mid \bar{Q}_{2}\right]$ in

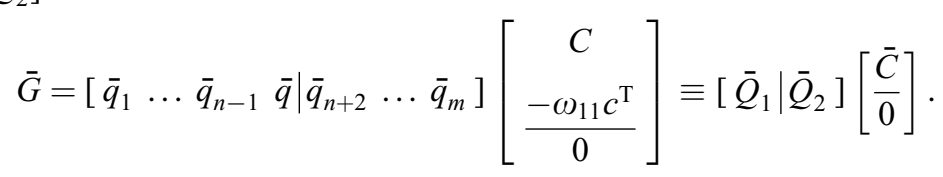

Here $\bar{C}$ is $n \times n$ nonsingular when $\omega_{11}>0$. For later use, (17) shows

$$
Q_{2} U_{1}=\left[\begin{array}{cc}
\omega_{11} & 0 \\
\tilde{q}_{n+1} & \bar{Q}_{2}
\end{array}\right] \text {. }
$$

Now look at the smallest singular value $\sigma_{\min }(\cdot)$ :

$$
\begin{aligned}
\sigma_{\min }(\bar{G})=\sigma_{\min }(\bar{C}) & =\min _{\left(u^{\mathrm{T}}, \mu\right) \neq 0}\left\|\left[C^{\mathrm{T}},-c \omega_{11}\right]\left[\begin{array}{l}
u \\
\mu
\end{array}\right]\right\|_{2} /\left\|\left[\begin{array}{l}
u \\
\mu
\end{array}\right]\right\|_{2} \\
& \leqslant\left\|c \omega_{11}\right\|_{2}=\frac{\omega_{11}\|g\|_{2}}{\sqrt{1-\omega_{11}^{2}}}
\end{aligned}
$$


(by setting $u=0$ ). Since $\|g\|=\left\|R^{\mathrm{T}} R^{-\mathrm{T}} g\right\| \leqslant\|R\| \cdot\left\|R^{-\mathrm{T}} g\right\|$ and $\omega_{11}=\sqrt{1-\left\|R^{-\mathrm{T}} g\right\|^{2}}$ (see (13)), we see that the bound (23) is tighter than the bound (14), and sometimes significantly so. It follows from (23) that for the condition number $\kappa_{2}(\bar{G})$ for solution of equations

$$
\kappa(\bar{G}) \equiv \frac{\sigma_{\max }(\bar{G})}{\sigma_{\min }(\bar{G})} \geqslant \frac{\sqrt{1-\omega_{11}^{2}}}{\omega_{11}} \frac{\|\bar{G}\|_{2}}{\|g\|_{2}},
$$

while for the error covariance $\sigma^{2}\left(\bar{G}^{\mathrm{T}} \bar{G}\right)^{-1}$ of the LS estimator $\bar{z}$ for this reduced model

$$
\|\operatorname{cov}\{\bar{z}-z\}\|_{2}=\sigma^{2}\left\|\left(\bar{G}^{\mathrm{T}} \bar{G}\right)^{-1}\right\|_{2}=\frac{\sigma^{2}}{\sigma_{\min }^{2}(\bar{G})} \geqslant \frac{\sigma^{2}\left(1-\omega_{11}^{2}\right)}{\omega_{11}^{2}\|g\|_{2}^{2}},
$$

where the largest semi-axis of the ellipsoid defined by the covariance matrix is the square root of $\|\operatorname{cov}\{\bar{z}-z\}\|_{2}$, that is $\sigma / \sigma_{\min }(\bar{G})$. So when the $i$ th observation and row of $G$ is deleted, small $\omega_{i i}$ leads to large error covariance and radials for the reduced model. Small enough $\omega_{i i}$ will result in good integrity algorithms rejecting this reduced model as being unable to support integrity. Clearly small $\omega_{i i}$ has severe negative consequences.

To maintain integrity we may have to consider deleting observations. But if the resulting reduced model has too bad error covariance, it will not be usable. Suppose for it to be acceptable requires of the largest semi-axis of the ellipsoid defined by the covariance matrix

$$
\text { Largest semi-axis } \leqslant \rho_{\max } .
$$

Then from (24) we see we cannot achieve this, and so cannot use the model obtained by deleting the $i$ th observation from (4), if

$$
\frac{\sigma \sqrt{1-\omega_{i i}^{2}}}{\omega_{i i}\left\|G^{\mathrm{T}} e_{i}\right\|_{2}}>\rho_{\max } .
$$

This emphasizes that small $\omega_{i i}$ both hides a possible fault and gives a poor (large) error covariance when the $i$ th observation is removed.

\section{A uniform derivation of test statistics}

Here, we use an orthogonalization technique to give a very uniform derivation of key statistics often used for fault detection and isolation, see for example Kelly (1998).

In order to test for and sometimes maintain integrity, we will have to delete possibly faulty observations from our linear model (4). We want to understand how the parity vector $p$ and residual $r$ behave in this case. The relevant equations are from (8) to (10)

$$
p \equiv Q_{2}^{\mathrm{T}} y, \quad r=Q_{2} p, \quad \omega_{i i}=\left\|e_{i}^{\mathrm{T}} Q_{2}\right\|_{2} .
$$

Since we are not dealing with $z$ in (4) here, we need only consider a less restrictive version of the $Q R$ factorization, one we call the pseudo- $Q R$ factorization, which for 
any $G$ satisfies

$$
G=\left[Q_{1} \mid Q_{2}\right]\left[\frac{R}{0}\right], \quad\left[Q_{1} \mid Q_{2}\right] \text { orthogonal, } R \text { full row rank }
$$

the difference being that $R$ need not be upper triangular (or upper trapezoidal if $G$ does not have full column rank). Here, as always 'orthogonal' means square with orthonormal columns, and the matrices are conformably partitioned. Clearly, the $Q R$ factorization can be found from the pseudo- $Q R$ factorization by carrying out the $Q R$ factorization of $R$. The pseudo- $Q R$ factorization is not unique, but every pseudo- $Q R$ factorization of $G$ will give the same $\|p\|_{2}, r$ and $\omega_{i i}$ in (26).

Without loss of generality we can consider deleting the first observation. When we delete this, we need the pseudo- $Q R$ factorization $\bar{G}=\bar{Q}_{1} \bar{R}$ where in (4)

$$
\bar{G}=\bar{Q}_{1} \bar{R}, \quad G=\left[\frac{g^{\mathrm{T}}}{\bar{G}}\right], \quad y=\left[\frac{\eta}{\bar{y}}\right] .
$$

Let $p_{1}$ and $r_{1}$ be the parity vector and residual for the reduced model $\bar{y}=\bar{G} z+\bar{v}+\bar{b}$, $\bar{v} \sim \mathrm{N}\left(0, \sigma^{2} I\right)$, see (4) and (5). Assume $m \times n G$ has full column rank. Let $U_{1}$ and $V_{1}$ be orthogonal matrices so that if (27) is the standard $Q R$ factorization of $G$,

$$
\begin{aligned}
& G=\left[Q_{1} V_{1} \mid Q_{2} U_{1}\right]\left[\frac{V_{1}^{\mathrm{T}} R}{0}\right] \equiv\left[\begin{array}{cc|cc}
0 & \gamma_{11} & \omega_{11} & 0 \\
\tilde{Q}_{1} & \tilde{q}_{n} & \tilde{q}_{n+1} & \tilde{Q}_{2}
\end{array}\right]\left[\begin{array}{c}
C \\
c^{\mathrm{T}} \\
\hline 0
\end{array}\right], \\
& \gamma_{11} \geqslant 0, \quad \omega_{11} \geqslant 0 .
\end{aligned}
$$

This is clearly a pseudo- $Q R$ factorization. Since $\left[Q_{1} V_{1} \mid Q_{2} U_{1}\right]$ has unit length columns and rows

$$
\gamma_{11}^{2}+\omega_{11}^{2}=1, \quad \gamma_{11}^{2}+\left\|\tilde{q}_{n}\right\|_{2}^{2}=1 .
$$

There are two cases we must treat here, the first being so unlikely it is usually ignored, but it is necessary for rigor.

Case 1. $\omega_{11}=0$. (We can take $U_{1}=I$ in (29)). We see this implies $\gamma_{11}=1$ and $\tilde{q}_{n}=0$, so (29) becomes

$$
\left[\begin{array}{c}
g^{\mathrm{T}} \\
\bar{G}
\end{array}\right]=G=\left[\frac{c^{\mathrm{T}}}{\tilde{Q}_{1} C}\right], \quad \bar{G}=\bar{Q}_{1} \bar{R} \equiv \tilde{Q}_{1} C,
$$

giving the desired pseudo- $Q R$ factorization of $\bar{G}$. Note in this special case $\bar{Q}_{1}$ has one less column than $Q_{1}$, while $\bar{Q}_{2} \equiv\left[\tilde{q}_{n+1}, \tilde{Q}_{2}\right]$ has the same number of columns as $Q_{2}$. In this case, see (26) and (28),

$$
\begin{aligned}
& Q_{2}=\left[\begin{array}{c}
0 \\
\bar{Q}_{2}
\end{array}\right], \quad p_{1} \equiv \bar{Q}_{2}^{\mathrm{T}} \bar{y}=\left[0, \bar{Q}_{2}^{\mathrm{T}}\right]\left[\begin{array}{l}
\eta \\
\bar{y}
\end{array}\right]=Q_{2}^{\mathrm{T}} y=p, \\
& r_{1}=\bar{Q}_{2} p_{1}=\bar{Q}_{2} p, \quad r=Q_{2} p=\left[\begin{array}{c}
0 \\
\bar{Q}_{2}
\end{array}\right] p=\left[\begin{array}{c}
0 \\
r_{1}
\end{array}\right] .
\end{aligned}
$$


We see when $\omega_{i i}=0,\|p\|_{2}=\|r\|_{2}$ is not altered by deleting the $i$ th observation. In simple terms this is because when $\omega_{i i}=0, Q_{2}^{\mathrm{T}} e_{i}=0$, see (26), so the $i$ th element of $y$ does not contribute to the parity vector $p=Q_{2}^{\mathrm{T}} y$ or the residual $r=Q_{2} Q_{2}^{\mathrm{T}} y$, and when the $i$ th observation is removed, the parity vector is unchanged, and the zero $i$ th element of $r$ is removed, leaving what are now the elements of the new LS residual. Of course $p$ is arbitrary up to orthogonal $U$ in $U^{\mathrm{T}} p$, so we should really say $\|p\|_{2}$ is unchanged, rather than $p$.

Case 2. $\omega_{11}>0$. Consider the $2 \times 2$ rotation $\tilde{J}$, defined using the scalars in (29)

$$
\tilde{J} \equiv\left[\begin{array}{cc}
\gamma_{11} & -\omega_{11} \\
\omega_{11} & \gamma_{11}
\end{array}\right], \quad \tilde{J}^{\mathrm{T}}\left[\begin{array}{l}
c^{\mathrm{T}} \\
0
\end{array}\right]=\left[\begin{array}{c}
\gamma_{11} c^{\mathrm{T}} \\
-\omega_{11} c^{\mathrm{T}}
\end{array}\right], \quad\left[\begin{array}{cc}
\gamma_{11} & \omega_{11} \\
\tilde{q}_{n} & \tilde{q}_{n+1}
\end{array}\right] \tilde{J}=\left[\begin{array}{cc}
1 & 0 \\
0 & \bar{q}
\end{array}\right],
$$

where the zero $(2,1)$ submatrix of the rightmost matrix follows since each column has unit length. Let $J_{n, n+1} \equiv \operatorname{diag}\left(I_{n-1}, \tilde{J}, I_{m-n-1}\right)$, and apply $J_{n, n+1} J_{n, n+1}^{\mathrm{T}}$ between the two factors of $G$ in (29) to give

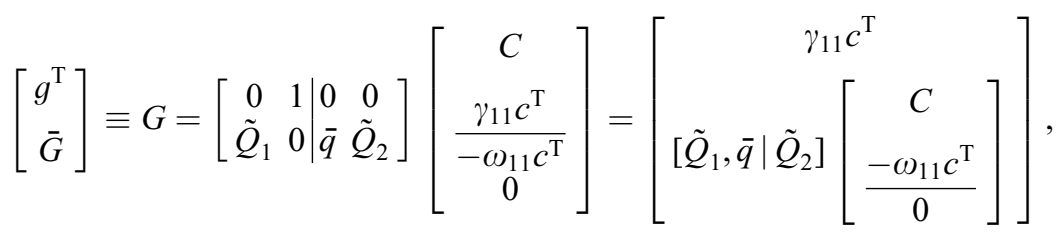

$$
\begin{aligned}
& \bar{G}=\left[\bar{Q}_{1} \mid \bar{Q}_{2}\right]\left[\begin{array}{c}
\bar{R} \\
0
\end{array}\right], \quad \bar{Q}_{1} \equiv\left[\tilde{Q}_{1}, \bar{q}\right], \quad \bar{Q}_{2} \equiv \tilde{Q}_{2}, \quad \bar{R} \equiv\left[\begin{array}{c}
C \\
-\omega_{11} c^{\mathrm{T}}
\end{array}\right] \text {, }
\end{aligned}
$$

giving the pseudo- $Q R$ factorization of $\bar{G}$. Here $\bar{R}=\operatorname{diag}\left(I_{n-1},-\omega_{11}\right) V_{1}^{\mathrm{T}} R$, see (29), and so has full row rank.

With our choice of $U_{1}$ giving $Q_{2} U_{1}$ in (29), define

$$
w_{1} \equiv\left[\begin{array}{c}
\omega_{11} \\
\tilde{q}_{n+1}
\end{array}\right]=Q_{2} U_{1} e_{1}, \quad \delta_{1} \equiv y^{\mathrm{T}} w_{1}=y^{\mathrm{T}} Q_{2} U_{1} e_{1}, \quad \tilde{p} \equiv U_{1}^{\mathrm{T}} p=U_{1}^{\mathrm{T}} Q_{2}^{\mathrm{T}} y,
$$

so that $\tilde{p}=U_{1}^{\mathrm{T}} Q_{2}^{\mathrm{T}} y$ is an allowable transformation of the full model parity vector $p=Q_{2}^{\mathrm{T}} y$, then from (29) and (33)

$$
\begin{aligned}
& \tilde{p}^{\mathrm{T}}=p^{\mathrm{T}} U_{1}=y^{\mathrm{T}} Q_{2} U_{1}=y^{\mathrm{T}}\left[\begin{array}{c|c}
\omega_{11} & 0 \\
\tilde{q}_{n+1} & \bar{Q}_{2}
\end{array}\right]=\left[\delta_{1} \mid \bar{y}^{\mathrm{T}} \bar{Q}_{2}\right]=\left[\delta_{1} \mid p_{1}^{\mathrm{T}}\right], \\
& \|p\|_{2}^{2}=\|\tilde{p}\|_{2}^{2}=\left\|p_{1}\right\|_{2}^{2}+\delta_{1}^{2}, \\
& \delta_{1}=\omega_{11} \eta+\tilde{q}_{n+1}^{\mathrm{T}} \bar{y}, \quad\left|\delta_{1}\right| \leqslant \omega_{11}|\eta|+\sqrt{1-\omega_{11}^{2}}\|\bar{y}\|_{2},
\end{aligned}
$$

(see (28) for the definition of $\eta$ and $\bar{y}$ ) since from (29) $1=\omega_{11}^{2}+\left\|\tilde{q}_{n+1}\right\|_{2}^{2}$. Clearly, $\|p\|_{2}=\|r\|_{2}$ cannot increase when an observation is deleted. Note that if we delete all but $n$ observations, and the remaining $\bar{G}$ is nonsingular, then the residual is zero and the parity vector nonexistent, so both of these can decrease to zero. 
This completes the two cases for the change in $\|p\|_{2}=\|r\|_{2}$ when an observation is deleted. Now we consider the problem of determining which measurement to delete. Since a faulty observation would probably lead to a large residual, one obvious criterion is to delete the measurement that leads to the greatest decrease in $\|p\|_{2}=\|r\|_{2}$. Let $p_{i}$ and $r_{i}$ be the parity vector and LS residual for the model (4) with $i$ th observation deleted. If $\omega_{i i}=0$ we saw deleting the $i$ th observation left $\|p\|_{2}$ unchanged-the worst possible result (since we seek the maximum decrease), so the criterion would generally not choose this, but as we will see below, it is still useful to know what effect it would have. When $\omega_{i i}>0$ we can choose orthogonal $U_{i}$ similar to the way $U_{1}$ was chosen in (29), to give $e_{i}^{\mathrm{T}} Q_{2} U_{i}=\omega_{i i} e_{1}^{\mathrm{T}}$, and define $\delta_{i}$ similar to the way $\delta_{1}$ was defined in (34), giving

$$
\begin{aligned}
& Q_{2} U_{i}=\left[\begin{array}{c|c}
\times & \times \\
\omega_{i i} & 0 \\
\times & \times
\end{array}\right] \equiv\left[\begin{array}{c|c}
w_{i} & 0 \\
& \times
\end{array}\right], \quad \delta_{i} \equiv y^{\mathrm{T}} Q_{2} U_{i} e_{1}=y^{\mathrm{T}} w_{i} \\
& \tilde{p}^{\mathrm{T}}=p^{\mathrm{T}} U_{i}=y^{\mathrm{T}} Q_{2} U_{i}=y^{\mathrm{T}}\left[\begin{array}{c}
w_{i} \\
0 \\
\times
\end{array}\right]=\left[\delta_{i} \mid p_{i}^{\mathrm{T}}\right], \quad\left\|p_{i}\right\|_{2}^{2}=\|p\|_{2}^{2}-\delta_{i}^{2}
\end{aligned}
$$

so deleting the $i$ th observation leads to a decrease in $\|p\|_{2}^{2}$ of $\delta_{i}^{2}$, and $i$ giving the greatest decrease in $\|p\|_{2}=\|r\|_{2}$ is that for which $\left|\delta_{i}\right|$ is greatest for those $\omega_{i i}>0$. Clearly the maximum $\left|\delta_{i}\right|$ gives the greatest decrease in $\|r\|_{2}$ and $\|p\|_{2}$.

It follows, in general, that

$$
\rho_{i} \equiv e_{i}^{\mathrm{T}} r=e_{i}^{\mathrm{T}} Q_{2} Q_{2}^{\mathrm{T}} y=e_{i}^{\mathrm{T}} Q_{2} U_{i} U_{i}^{\mathrm{T}} Q_{2}^{\mathrm{T}} y=\omega_{i i} e_{1}^{\mathrm{T}} U_{i}^{\mathrm{T}} Q_{2}^{\mathrm{T}} y=\omega_{i i} \delta_{i} .
$$

Thus when $\omega_{i i} \neq 0$, the $\delta_{i}$ could be obtained directly from $\delta_{i}=\rho_{i} / \omega_{i i}$ without computing the $U_{i}$.

We summarize the above as a theorem.

Theorem 4.1. Let $p$ be the parity vector as in (8), $\rho_{i}$ be the ith element of the LS residual $r$ in (9), and let $p_{i}$ and $r_{i}$ be the parity vector and LS residual for the model (4) with ith observation deleted. If $\omega_{i i} \equiv\left\|Q_{2}^{\mathrm{T}} e_{i}\right\|_{2}=0$ in (10), then

$$
\|p\|_{2}-\left\|p_{i}\right\|=\|r\|_{2}-\left\|r_{i}\right\|_{2}=0 .
$$

If $\omega_{i i}>0$ and $U_{i}$ is chosen so that $e_{i}^{\mathrm{T}} Q_{2} U_{i}=\omega_{i i} e_{1}^{\mathrm{T}}$, then with $\delta_{i} \equiv e_{1}^{\mathrm{T}} U_{i}^{\mathrm{T}} Q_{2}^{\mathrm{T}} y, \delta_{i}=\rho_{i} / \omega_{i i}$ and

$$
\left|\delta_{i}\right|=\sqrt{\|r\|_{2}^{2}-\left\|r_{i}\right\|_{2}^{2}}=\sqrt{\|p\|_{2}^{2}-\left\|p_{i}\right\|_{2}^{2}}=\left|\rho_{i}\right| / \omega_{i i} \leqslant \omega_{i i}|\eta|+\sqrt{1-\omega_{i i}^{2}}\|\bar{y}\|_{2},
$$

where $\bar{y}$ is $y$ with its ith element $\eta$ removed.

There is a lack of uniqueness in the definition of $\delta_{i}$ in (36). $\delta_{i}$ is uniquely defined while $\omega_{i i}>0$, but when $\omega_{i i}=0, U_{i}$ can be any orthogonal matrix, and we can take $U_{i}$ in (37) to give any value of $\delta_{i}$ in $-\|p\|_{2} \leqslant \delta_{i} \leqslant\|p\|_{2}$. Similarly in (38) $\omega_{i i}=0 \Rightarrow$ $\rho_{i}=0$ and $\delta_{i}$ is not defined. To remove this lack of uniqueness we could define $\delta_{i}^{2}$ 
to be the decrease in $\|p\|_{2}^{2}$ when the $i$ th observation is removed, and then (31) shows $\omega_{i i}=0 \Rightarrow \delta_{i}=0$.

Here we need to point out that $\delta_{i}=y^{\mathrm{T}} Q_{2} U_{i} e_{1}$ is the statistic used in Brenner (1990) for fault detection, $\delta_{i}^{2}=\|r\|_{2}^{2}-\left\|r_{i}\right\|_{2}^{2}$ is the statistic presented in Parkinson and Axelrad (1988) for fault isolation, and $\delta_{i}=\rho_{i} / \omega_{i i}$ is the statistic proposed in Sturza (1988-89) for fault isolation. The equivalence of the three quantities has been realized by Kelly (1998). But our proof here is a uniform approach and is simpler, and the case that $\omega_{i i}=0$ is not discussed in these papers.

Note if there is no fault, i.e., $b=0$, then from (9) and (10) we see $\delta_{i}=\rho_{i} / \omega_{i i}$ has a normal distribution $\mathrm{N}\left(0, \sigma^{2}\right)$. Since we do not know which satellite may have a fault, we have to check all individual observations for a potential fault. There are a total of $m$ statistics $\delta_{i}, i=1, \ldots, m$. In Kelly (1998), it is suggested to use $\max _{i}\left|\delta_{i}\right|$ for fault detection and isolation. The value of $\max _{i}\left|\delta_{i}\right|$ has to be compared with a threshold, which can be derived by using a given false detection probability. If the former is larger than the latter, we will say that a fault has occurred and identify the corresponding satellite which makes $\left|\delta_{i}\right|$ maximum as faulty.

\section{Sensitivity of the test statistics}

In the last section, the quantity $\delta_{i}$ is used for fault detection or isolation. In addition to that, the following quantity is also often used in the literature as a statistic for fault detection (see, for example, Sturza, 1988-89):

$$
\delta=r^{\mathrm{T}} r \text {. }
$$

We see from (8) and (9) that $\delta=p^{\mathrm{T}} p$, and if there is no fault with any satellite, then with (4) $p \sim \mathrm{N}\left(0, \sigma^{2} I_{m-n}\right)$, so $\delta / \sigma^{2}$ will have a $\chi^{2}$ distribution.

Since usually the given data $G$ and $y$ in the model (4) are not known exactly, we would like to know how small relative errors in the data contribute to errors in the statistics. Since the absolute values of the statistics are compared to the corresponding thresholds when we do fault detection or isolation tests, we are only interested in the absolute errors in the statistics caused by errors in the data.

In our analysis we assume $\omega_{i i} \neq 0$. Suppose the $m \times n$ matrix $G(t)$ is a continuous differentiable function of $t$ with full column rank for $|t| \leqslant \theta$ and $G(0)=G$, and suppose $y(t)$ is also a continuously differentiable function of $t$ and $y(0)=y$. Then we can define the $Q R$ factorization of $G(t)$ and the test statistics $\delta(t)$ and $\delta_{i}(t)$, where we assume $\theta$ is small enough such that $\omega_{i i}(t) \neq 0$ for $|t| \leqslant \theta$. For any matrix (vector or scalar) $A$, denote $\dot{A} \equiv\left(\mathrm{d} a_{i j}(t) /\left.\mathrm{d} t\right|_{t=0}\right)$. Since $G(t)=Q_{1}(t) R(t)$ for $|t| \leqslant \theta$, we have

$$
\dot{G}=\dot{Q}_{1} R+Q_{1} \dot{R} \text {. }
$$

Then it follows that

$$
\dot{Q}_{1}=\dot{G} R^{-1}-Q_{1} \dot{R} R^{-1} \text {. }
$$

From $G(t)^{\mathrm{T}} G(t)=R(t)^{\mathrm{T}} R(t)$, it follows that

$$
\dot{G}^{\mathrm{T}} G+G^{\mathrm{T}} \dot{G}=\dot{R}^{\mathrm{T}} R+R^{\mathrm{T}} \dot{R} .
$$


Therefore

$$
R^{-\mathrm{T}} \dot{G}^{\mathrm{T}} Q_{1}+Q_{1}^{\mathrm{T}} \dot{G} R^{-1}=R^{-\mathrm{T}} \dot{R}^{\mathrm{T}}+\dot{R} R^{-1} .
$$

Then from (41), (42) and $I-Q_{1} Q_{1}^{\mathrm{T}}=Q_{2} Q_{2}^{\mathrm{T}}$ we obtain for later use

$$
\begin{aligned}
\dot{Q}_{1} Q_{1}^{\mathrm{T}}+Q_{1} \dot{Q}_{1}^{\mathrm{T}} & =\dot{G} R^{-1} Q_{1}^{\mathrm{T}}-Q_{1} \dot{R} R^{-1} Q_{1}^{\mathrm{T}}+Q_{1} R^{-\mathrm{T}} \dot{G}^{\mathrm{T}}-Q_{1} R^{-\mathrm{T}} \dot{R}^{\mathrm{T}} Q_{1}^{\mathrm{T}} \\
& =Q_{2} Q_{2} \dot{G} R^{-1} Q_{1}^{\mathrm{T}}+Q_{1} R^{-\mathrm{T}} \dot{G}^{\mathrm{T}} Q_{2} Q_{2}^{\mathrm{T}} .
\end{aligned}
$$

First we discuss the sensitivity of $\delta$. Note that

$$
r(t)^{\mathrm{T}} r(t)=y(t)^{\mathrm{T}} Q_{2}(t) Q_{2}(t)^{\mathrm{T}} y(t)=y(t)^{\mathrm{T}}\left(I-Q_{1}(t) Q_{1}(t)^{\mathrm{T}}\right) y(t) .
$$

So we have with (43)

$$
\begin{aligned}
\dot{\delta} & =\dot{y}^{\mathrm{T}} Q_{2} Q_{2}^{\mathrm{T}} y+y^{\mathrm{T}} Q_{2} Q_{2}^{\mathrm{T}} \dot{y}-y^{\mathrm{T}}\left(\dot{Q}_{1} Q_{1}^{\mathrm{T}}+Q_{1} \dot{Q}_{1}^{\mathrm{T}}\right) y \\
& =\dot{y}^{\mathrm{T}} Q_{2} Q_{2}^{\mathrm{T}} y+y^{\mathrm{T}} Q_{2} Q_{2}^{\mathrm{T}} \dot{y}-y^{\mathrm{T}}\left(Q_{2} Q_{2}^{\mathrm{T}} \dot{G} R^{-1} Q_{1}^{\mathrm{T}}+Q_{1} R^{-\mathrm{T}} \dot{G}^{\mathrm{T}} Q_{2} Q_{2}^{\mathrm{T}}\right) y .
\end{aligned}
$$

It follows since $\|y\|^{2}=\|r\|^{2}+\left\|Q_{1}^{\mathrm{T}} y\right\|^{2}$, see (9) with (7), that

$$
\begin{aligned}
|\dot{\delta}| & \leqslant 2\left\|Q_{2}^{\mathrm{T}} y\right\|_{2}\left\|Q_{2}^{\mathrm{T}} \dot{y}\right\|_{2}+2\left\|R^{-1}\right\|_{2}\left\|Q_{1}^{\mathrm{T}} y\right\|_{2}\left\|Q_{2}^{\mathrm{T}} y\right\|_{2}\left\|Q_{2}^{\mathrm{T}} \dot{G}\right\|_{2} \\
& =2\|y\|_{2}\|r\|_{2} \frac{\left\|Q_{2}^{\mathrm{T}} \dot{y}\right\|_{2}}{\|y\|_{2}}+2\|r\|_{2} \sqrt{\|y\|_{2}^{2}-\|r\|_{2}^{2}} \kappa_{2}(G) \frac{\left\|Q_{2}^{\mathrm{T}} \dot{G}\right\|_{2}}{\|G\|_{2}} .
\end{aligned}
$$

We see that if $\dot{y} \in \mathscr{R}(G)$ and $\mathscr{R}(\dot{G}) \subseteq \mathscr{R}(G)$, then $\dot{\delta}=0$. The crucial factor in the bound above is $\|r\|_{2} \sqrt{\|y\|_{2}^{2}-\|r\|_{2}^{2}} \kappa_{2}(G)$. If this is large, then small relative errors in $G$ may result in big errors in $\delta$.

Now we do a sensitivity analysis of the statistic $\delta_{i}$. From $\delta_{i}(t)=e_{i}^{\mathrm{T}} r(t) / \omega_{i i}(t)$ we have

$$
\dot{\delta}_{i}=\frac{e_{i}^{\mathrm{T}} \dot{r}}{\omega_{i i}}-\frac{e_{i}^{\mathrm{T}} r \dot{\omega}_{i i}}{\omega_{i i}^{2}} .
$$

Since $r=Q_{2} Q_{2}^{\mathrm{T}} y=\left(I-Q_{1} Q_{1}^{\mathrm{T}}\right) y$, it follows that

$$
\dot{r}=Q_{2} Q_{2}^{\mathrm{T}} \dot{y}-\left(\dot{Q}_{1} Q_{1}^{\mathrm{T}}+Q_{1} \dot{Q}_{1}^{\mathrm{T}}\right) y .
$$

Since $\omega_{i i}^{2}=e_{i}^{\mathrm{T}} Q_{2} Q_{2}^{\mathrm{T}} e_{i}=e_{i}^{\mathrm{T}}\left(I-Q_{1} Q_{1}^{\mathrm{T}}\right) e_{i}$, it follows that

$$
\dot{\omega}_{i i}=-\frac{e_{i}^{\mathrm{T}}\left(\dot{Q}_{1} Q_{1}^{\mathrm{T}}+Q_{1} \dot{Q}_{1}^{\mathrm{T}}\right) e_{i}}{2 \omega_{i i}} .
$$

Then from (45) to (47) and using (43), we obtain

$$
\begin{aligned}
\dot{\delta}_{i}= & \frac{e_{i}^{\mathrm{T}} Q_{2} Q_{2}^{\mathrm{T}} \dot{y}-e_{i}^{\mathrm{T}}\left(Q_{2} Q_{2}^{\mathrm{T}} \dot{G} R^{-1} Q_{1}^{\mathrm{T}}+Q_{1} R^{-\mathrm{T}} \dot{G}^{\mathrm{T}} Q_{2} Q_{2}^{\mathrm{T}}\right) y}{\omega_{i i}} \\
& +\frac{e_{i}^{\mathrm{T}} Q_{2} Q_{2}^{\mathrm{T}} y e_{i}^{\mathrm{T}}\left(Q_{2} Q_{2}^{\mathrm{T}} \dot{G} R^{-1} Q_{1}^{\mathrm{T}}+Q_{1} R^{-\mathrm{T}} \dot{G}^{\mathrm{T}} Q_{2} Q_{2}^{\mathrm{T}}\right) e_{i}}{2 \omega_{i i}^{3}} .
\end{aligned}
$$


Notice that $\left\|Q_{2}^{\mathrm{T}} e_{i}\right\|_{2}=\omega_{i i}$ and $\left\|Q_{1}^{\mathrm{T}} e_{i}\right\|_{2}=\sqrt{1-\omega_{i i}^{2}}$, so we have

$$
\begin{aligned}
\left|\dot{\delta}_{i}\right| \leqslant & \left\|Q_{2}^{\mathrm{T}} \dot{y}\right\|_{2}+\left\|R^{-1}\right\|_{2}\left\|Q_{1}^{\mathrm{T}} y\right\|_{2}\left\|Q_{2}^{\mathrm{T}} \dot{G}\right\|_{2}+\frac{\sqrt{1-\omega_{i i}^{2}}}{\omega_{i i}}\left\|R^{-1}\right\|\left\|Q_{2}^{\mathrm{T}} y\right\|_{2}\left\|Q_{2}^{\mathrm{T}} \dot{G}\right\|_{2} \\
& +2 \frac{\sqrt{1-\omega_{i i}^{2}}}{2 \omega_{i i}}\left\|R^{-1}\right\|\left\|Q_{2}^{\mathrm{T}} y\right\|_{2}\left\|Q_{2}^{\mathrm{T}} \dot{G}\right\|_{2} \\
= & \|y\|_{2} \frac{\left\|Q_{2}^{\mathrm{T}} \dot{y}\right\|_{2}}{\|y\|_{2}}+\left(\sqrt{\|y\|_{2}^{2}-\|r\|_{2}^{2}}+\frac{2 \sqrt{1-\omega_{i i}^{2}}}{\omega_{i i}}\|r\|_{2}\right) \kappa_{2}(G) \frac{\left\|Q_{2}^{\mathrm{T}} \dot{G}\right\|_{2}}{\|G\|_{2}} .
\end{aligned}
$$

Like $\delta, \delta_{i}=0$ if $\dot{y} \in \mathscr{R}(G)$ and $\mathscr{R}(\dot{G}) \subseteq \mathscr{R}(G)$. The crucial factor in the bound above is $\left(\sqrt{1-\omega_{i i}^{2}} / \omega_{i i}\right)\|r\|_{2} \kappa_{2}(G)$. Note if $\omega_{i i}$ is small, then small relative errors in $G$ may lead to large errors in $\delta_{i}$. Again we see that a small $\omega_{i i}$ has a damaging effect. However, a small $\omega_{i i}$ does not seem to make the statistic $\delta$ in (40) more sensitive, see (44).

\section{Summary}

Some basic GPS integrity theory has been presented. We focused largely on the "fault observability" scalars $\omega_{i i}$, and discussed the damaging effect of a small or zero $\omega_{i i}$ on GPS integrity. An orthogonal transformation approach was used to derive three typical equivalent statistics for fault detection and isolation, and a sensitivity analysis of the test statistics was given. Once again $\omega_{i i}$ were seen to be of great theoretical and practical importance. Further computations would be required to compute the radials, but it can be shown that these also depend directly on $\omega_{i i}^{-1}$, and it is important to understand the fundamental nature of these $\omega_{i i}$ in the integrity theory of GPS.

\section{Acknowledgements}

We would like to thank a referee for pointing out the paper by Stewart (1979) and its relationship with some of the work here, see the last two paragraphs of Section 3.2.

\section{References}

Brenner, M., 1990. Implementation of a RAIM Monitor in a GPS Receiver and an Integrated GPS/IRS. Proceedings of the Third International Technical Meeting of the Satellite Division of the Institute of Navigation Meeting (ION GPS-90), September 19-21, Colorado Springs, CO, pp. 397-406.

Chang, X.-W., Paige, C.C., Studenny, J., 2000. Two Carrier Phase Based Approaches for Autonomous Fault Detection and Exclusion. Proceedings of the 13th International Technical Meeting of the Satellite Division of the Institute of Navigation (ION GPS 2000), September 19-22, Salt Lake City, Utah, pp. 1895-1905.

Chin, G.Y., Kraemer, J.H., Brown, R.G., 1992-93. GPS RAIM: screen out bad geometries under worst-case bias conditions. Navigation J. Inst. Navigation 39 (4), 407-428.

Cook, D., Weisberg, S., 1982. Residual and Influence in Regression. Chapman \& Hall, London.

Golub, G.H., Van Loan, C.F., 1996. Matrix Computations, 3rd Edition. The Johns Hopkins University Press, Baltimore, MD. 
Hofmann-Wellenhof, B., Lichtenegger, H., Collins, J., 1997. GPS Theory and Practice, 4th Edition. Springer, New York.

Kelly, R.J., 1998. The Linear Model, RNP, and the Near-Optimum Fault Detection and Exclusion Algorithm. Global Positioning System, Vol. V. The Institute of Navigation, pp. 227-259.

Lee, Y., Van Dyke, K., Decleene, B., Studenny, J., Beckmann, M., 1996. Summary of RTCA SC-159 GPS Integrity Working Group Activities. Navigation J. Inst. Navigation 43 (3), 307-325.

Parkinson, B.W., Axelrad, P., 1988. Autonomous GPS integrity monitoring using the pseudorange residual. Navigation J. Inst. Navigation 35 (2), 255-274.

Parkinson, B.W., Spilker, J.J., Axelrad, P., Enge, P. (Ed). 1996. Global Positioning System: Theory and Applications, Vol. 1 and Vol. 2. American Institute of Aeronautics and Astronautics, Inc., New York.

Stewart, G.W., 1979. The Effects of Rounding Error on an Algorithm for Downdating a Cholesky Factorization. J. Inst. Math. Appl. 23, 203-213.

Strang, G., Borre, K., 1997. Linear Algebra, Geodesy, and GPS. Wellesley-Cambridge Press, Wellesley, MA. Sturza, M.A., 1988-89. Navigation system integrity monitoring using redundant measurements. Navigation J. Inst. Navigation 35 (4), 483-501. 